AperTO - Archivio Istituzionale Open Access dell'Università di Torino

\title{
Characterization of the B-Center Defect in Diamond through the Vibrational Spectrum: A Quantum-Mechanical Approach
}

\section{This is the author's manuscript}

Original Citation:

Availability:

This version is available http://hdl.handle.net/2318/1663813

since 2018-03-26T14:21:54Z

Published version:

DOI:10.1021/acs.jpca.7b11551

Terms of use:

Open Access

Anyone can freely access the full text of works made available as "Open Access". Works made available under a Creative Commons license can be used according to the terms and conditions of said license. Use of all other works requires consent of the right holder (author or publisher) if not exempted from copyright protection by the applicable law. 


\title{
Characterization of the B-center Defect in
}

\section{Diamond Through the Vibrational Spectrum. A Quantum Mechanical Approach.}

Simone Salustro, ${ }^{* \dagger}$ Anna Maria Ferrari, ${ }^{\dagger}$ Francesco Silvio Gentile, ${ }^{\ddagger}$ Jacques Kontak Desmarais, ${ }^{\ddagger}$ Michel Rérat, $₫$ and Roberto Dovesi ${ }^{\dagger}$

$\dagger$ Dipartimento di Chimica, Università di Torino and NIS (Nanostructured Interfaces and Surfaces) Centre, Via P. Giuria 5, 10125 Torino, Italy

$\ddagger$ Dipartimento di Chimica, Università di Torino, Via P. Giuria 5, 10125 Torino, Italy

\Equipe de Chimie Physique, IPREM UMR5254, Université de Pau et des Pays de l'Adour, 64000 Pau (France)

E-mail: simone.salustro@unito.it

December 9, 2017

\begin{abstract}
The B-center in diamond, that consists of a vacancy whose 4 first nearest neighbors are nitrogen atoms, has been investigated at the quantum mechanical level with an all electron Gaussian type basis set, hybrid functionals and the periodic supercell approach. In order to simulate various defect concentrations, four cubic supercells have been considered, containing (before the creation of the vacancy) 64, 216, 512 and 1000 atoms, respectively. Whereas the B-center does not affect the Raman spectrum of diamond, several intense peaks appear in the IR spectrum, that should permit to identify this defect. It turns out that, of the seven peaks proposed by Sutherland in 1954, located at
\end{abstract}


$328,780,1003,1171,1332,1372$ and $1426 \mathrm{~cm}^{-1}$ and frequently mentioned as fingerprints of the B center, the first one and the last three do not appear in the simulated spectrum at any concentration. The graphical animation of the modes confirms the attribution of the remaining three, and permits also to investigate the nature of the full set of modes.

\section{Introduction}

Despite the simplicity of the pristine lattice and the very strong interaction between carbon atoms, both natural and synthetic diamonds are characterized by a large variety of pointdefects $^{1-6}$ that have attracted large theoretical and experimental interest. ${ }^{3-16}$ Among the large variety of experimental techniques, Infrared (IR) and Raman spectroscopies represent ideal methods in the attempt to characterize the atomic nature of the various point-defects in diamond-like materials. ${ }^{4,5,10,14,15,17-23}$

In a series of previous works, some of the present authors have proposed the quantum mechanical characterization of different point defects: ${ }^{24-29}$ vacancies, interstitials, combinations of the two and nitrogen inclusions. Very recently the A-center, the most common extrinsic defect in diamond, consisting of two first neighbors substitutional nitrogen atoms, has been investigated. ${ }^{29}$ In particular the IR spectrum has been generated at various defect concentrations (using supercells up to 216 atoms), with a very satisfactory agreement with the data reported by Sutherland et al. ${ }^{1}$ and the spectrum published by Davies. ${ }^{2}$

In this paper we focus our attention on another very common extrinsic defects: the B-center. According to Loubser and Van Wyk ${ }^{30}$ this defect has tetrahedral symmetry and consists of a vacancy whose four first neighbors are nitrogen atoms. These atoms are three-fold coordinated and lead to a closed-shell system.

Sutherland, ${ }^{1}$ in his seminal paper dating back to 1954, investigated 13 diamond samples of different origin, and identified 7 fundamental peaks possibly associated to the B-center at $328,780,1003,1171,1332,1372$ and $1426 \mathrm{~cm}^{-1}$. According to subsequent analyses, performed 20 to 30 years later (see, among others, Ref. 31-37) the first and the last two 
peaks were attributed to platelets; the peak at $1332 \mathrm{~cm}^{-1}$ is the main pure diamond feature; an additional peak was finally attributed to the B-defect, that seems then characterized by peaks at 780, 1003, 1098 and $1171 \mathrm{~cm}^{-1}$.

With regard to simulation, defects in diamond have been investigated by using both finite cluster and periodic supercell approaches, ${ }^{3,16,38-41}$ and (in most of the cases) using the simplest formulations of DFT (i.e. LDA or PBE). These approaches are, however, known to poorly describe the exchange interaction and to largely underestimate the band gaps in which the defect states are located.

Here we investigate the B-center by using the supercell scheme, as implemented in the CRYSTAL code, ${ }^{42}$ a local gaussian-type basis set and hybrid functionals. Following the same strategy adopted in a series of previous works, ${ }^{24-29}$ the structural, electronic and vibrational characterization of the defect is presented. As our previous study on the A-center ${ }^{29}$ has shown that the shape and the size of the supercell may play a key-role on the vibrational modes that largely coincide with the ideal diamond ones (modes below $1332 \mathrm{~cm}^{-1}$ ), much larger supercells have been considered than for the A-defect, in order to approach as much as possible the limit of the isolated defect. The four cells here considered are cubic $(a=b=c$, and $\alpha=\beta=\gamma=90^{\circ}$ ) and contain (before the creation of the vacancy) $64,216,512$ and 1000 atoms.

\section{Computational Details}

Calculations have been performed by using the B3LYP global hybrid functional, ${ }^{43,44}$ as implemented in the CRYSTAL program. ${ }^{42}$ Additional calculations on total energies have been repeated with the Hartree-Fock (HF) method and with other formulations of DFT, ranging from pure $\left(\mathrm{LDA}^{45}\right.$ and $\left.\mathrm{PBE}^{46}\right)$, to global $\left(\mathrm{PBE0}^{47}\right)$ and range-separated (HSE06 ${ }^{48}$ ) hybrids. An all-electron basis set of Gaussian-type functions has been adopted (Pople's 6-21G ${ }^{49}$ ). The exponent of the most diffuse $s p$ shell is 0.228 and $0.300 \mathrm{Bohr}^{-2}$ for $\mathrm{C}$ and $\mathrm{N}$, respec- 
tively. The defect formation energy has been calculated also with larger basis sets: $6-21 \mathrm{G}^{*}$, 6-31G and $6-31 \mathrm{G}^{*} .{ }^{50}$ The truncation criteria of the Coulomb and exchange infinite series are controlled by five thresholds, $\mathrm{T}_{i}$, which have been set to $8\left(\mathrm{~T}_{1}-\mathrm{T}_{4}\right)$ and $16\left(\mathrm{~T}_{5}\right)$. The convergence threshold on energy for the self-consistent-field (SCF) procedure has been set to $10^{-8}$ hartree for structural optimizations and to $10^{-10}$ hartree for vibration frequency calculations.

The DFT exchange-correlation contribution and its gradient are evaluated by numerical integration over the unit cell volume. The generation of the integration grid points in CRYSTAL is based on an atomic partition method, originally developed by Becke ${ }^{51}$ for molecules and furtherly extended to periodic systems. In this study the default ${ }^{52}$ pruned grid with 75 radial and 974 angular points has been used, whose accuracy can be measured by comparing the integrated charge density in the largest supercell here considered (1000 atoms), $N_{i}=5998.0876$, with the total number of 5998 electrons in the unit cell.

A periodic supercell approach is used in order to simulate different defect concentrations. Supercells $\mathrm{S}_{n}$ have been considered, with $n=64,216,512$ or 1000 . Reciprocal space has been sampled using a $\Gamma$-centered regular Pack-Monkhorst ${ }^{53}$ and Gilat ${ }^{54}$ sub-lattices (controlled by the SHRINK keyword, see the CRYSTAL User's Manual ${ }^{52}$ ) with a "shrinking factor" $\left(\mathrm{IS}^{52}\right)$ equal to 4 for $\mathrm{S}_{64}$ and 2 for $\mathrm{S}_{216}, \mathrm{~S}_{512}$ and $\mathrm{S}_{1000}$, corresponding to a sampling over 35 and 4 k-points in the symmetry irreducible part of the First Brillouin Zone (FBZ), respectively. Harmonic phonon frequencies, $\omega_{p}$, at the $\Gamma$ point (i.e. at the center of the first Brillouin zone in reciprocal space) are obtained from the diagonalization of the mass-weighted Hessian matrix of the second energy derivatives with respect to atomic displacements $u:^{55-59}$

$$
W_{a i, b j}^{\Gamma}=\frac{H_{a i, b j}^{0}}{\sqrt{M_{a} M_{b}}} \quad \text { with } \quad H_{a i, b j}^{\mathbf{0}}=\left(\frac{\partial^{2} E}{\partial u_{a i}^{\mathbf{0}} \partial u_{b j}^{\mathbf{0}}}\right)
$$

where atoms $a$ and $b$ (with atomic masses $M_{a}$ and $M_{b}$ ) in the reference cell, $\mathbf{0}$, are displaced along the $i$-th and $j$-th Cartesian directions, respectively. Here these are computed 
as numerical first derivative of the analytical energy gradients. ${ }^{60}$ Integrated intensities for IR absorption $\mathcal{I}_{p}$ are computed for each mode $p$ by means of the mass-weighted effective-mode Born-charge vector $\vec{Z}_{p}{ }^{61,62}$ evaluated through an analytical CPHF/KS approach: ${ }^{63,64}$

$$
\mathcal{I}_{p} \propto\left|\vec{Z}_{p}\right|^{2}
$$

The relative Raman intensities of the peaks are also computed analytically through a similar scheme. ${ }^{65,66}$

\section{Results}

This section is divided in two parts: in the first one the geometry of the defect, the mannerin which the lone pairs on the $4 \mathrm{~N}$ atoms interact and the position of the defective levels in the large band gap of the diamond are presented. In the second part a detailed analysis of the simulated spectroscopic features of the B-center is reported.
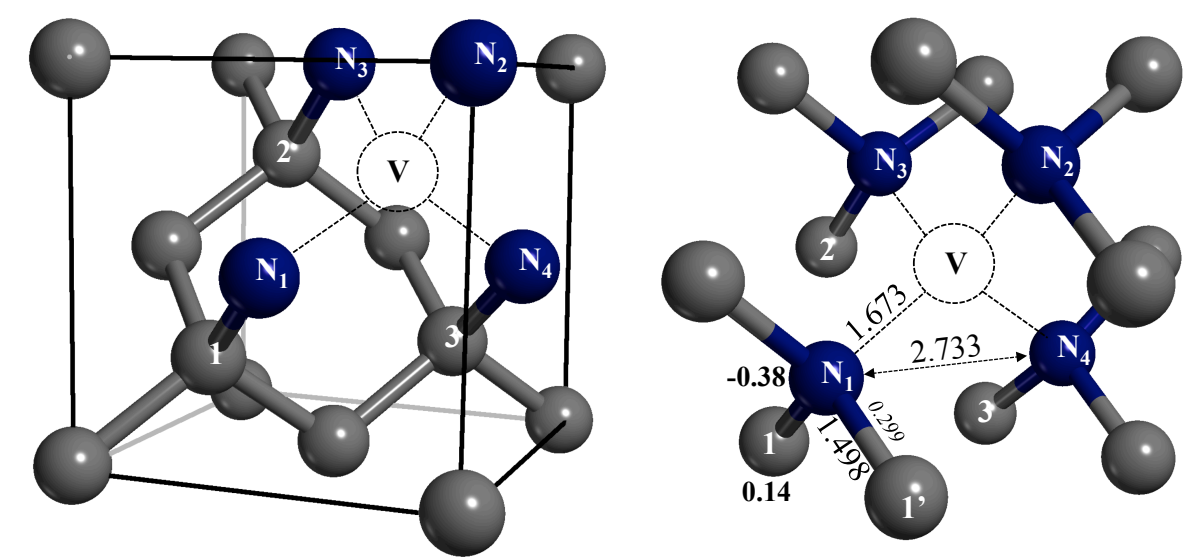

Figure 1: On the left side, the structure of the $\mathrm{B}$ defect (four $\mathrm{N}$ atoms surrounding a $\mathrm{C}$ vacancy) is shown. On the right side, the local cluster in its $T_{d}$ point symmetry is reported as well. Distances (in $\AA$, large), Mulliken charges (in $|e|$, bold) and bond populations (in $|e|$, italic) refer to the $\mathrm{S}_{216}$ supercell. The distance between nitrogen atoms and the centroid of the vacancy is $1.673 \AA$ (a similar value is observed for the isolated C vacancy in its $S_{z}=0$ state). 

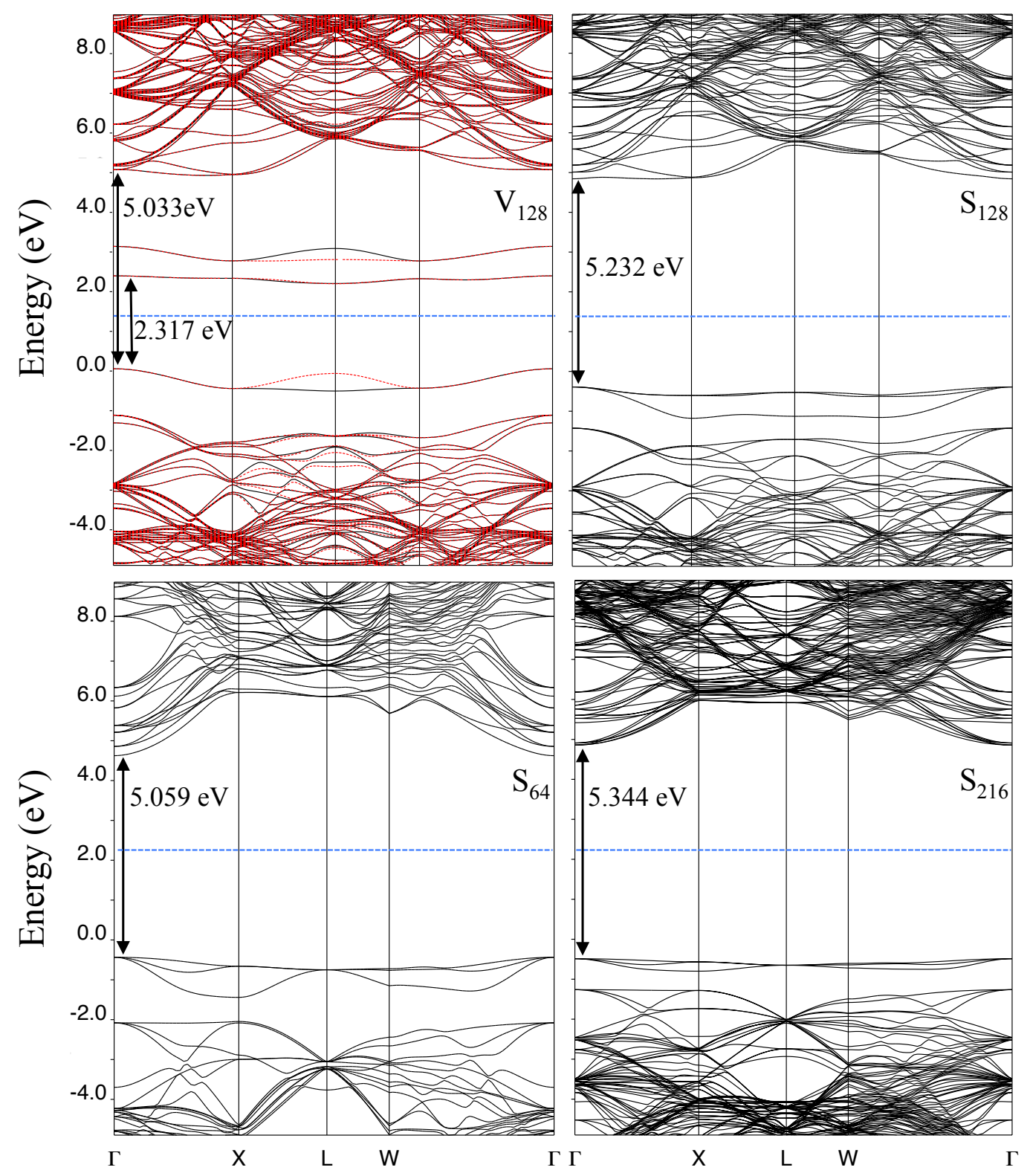

Figure 2: Band structures of the B-center at three defect concentrations. In the top left panel the band structure of the isolated vacancy in its most stable spin state $\left(\mathrm{S}_{z}=0\right)$ is reported for comparison: continous black lines represent the $\alpha$ energy levels, whereas dashed red lines represent the $\beta$ levels. With regard to the largest cells $\left(\mathrm{S}_{512}\right.$ and $\left.\mathrm{S}_{1000}\right)$, the energy difference between the defective levels and the lowest unoccupied level of diamond is 5.449 and 5.461 $\mathrm{eV}$, respectively. 


\section{Geometry and electron structure}

Together with the conventional cell of the defective structure (right), the local optimized geometry (from the $\mathrm{S}_{216}$ cell) in the vicinity of the B-center, as well as its charge distribution are reported in Figure 1. Since the mutual perturbation between defects hosted in neighboring cells goes rapidly to zero, the same values are observed also for the larger $\mathrm{S}_{512}$ and $\mathrm{S}_{1000}$ supercells. Each nitrogen atom forms three strong covalent bonds with the neighboring carbon atoms. The repulsive interaction among the lone pairs on each nitrogen atom, all oriented towards the vacant site, does not seem to affect the distance between the vacancy and its first nearest neighbors, which remains about the same as in the isolated vacancy $(\approx$ $1.67 \AA$ in both cases). The bond lenght between each nitrogen and its three neighboring carbon atoms is $1.498 \AA$ (the $\mathrm{C}$-C distance in diamond is $1.560 \AA$ ). The angle formed by the C-N bond with the N-V direction is about $106^{\circ}$, slightly smaller than the one of the regular tetrahedron. The higher electronegativity of nitrogen with respect to carbon leads to a strong polarization of the electron density, with Mulliken atomic net charges of $-0.38|e|$ for nitrogen, and $+0.14|e|$ on each of its three first neighboring carbons. The perturbation to the electron density introduced by the nitrogen atoms rapidly vanishes, being the Mulliken atomic net charge of their second neighbors as small as $0.01|e|$.

In Figure 2 the band structures of the B-center are reported for different sizes of the supercell. In the top two panels of the Figure a comparison between the band structure of the B-center and that of the isolated vacancy $(\mathrm{V})$ in its most stable state (singlet) is reported for $\mathrm{S}_{128}$. The energy levels of the four unpaired electrons surrounding the vacancy can easily be recognized in the wide band gap of diamond. The defective energy levels of the B-center are associated to 4 doubly occupied bands provided by the nitrogen lone pairs. One band, belonging to the totally symmetric irreducible representation $\left(\mathrm{A}_{1}\right)$, is a deep energy level, and is not visible in Figure 2. The remaining three defective levels belong to the $t_{2}$ irreducible representation, at $\Gamma$, and are about $0.80 \mathrm{eV}\left(\mathrm{S}_{216}\right)$ above the "pristine diamond" valence band. Concerning the effect of defect concentration, the comparison between the $\mathrm{S}_{64}$ and $\mathrm{S}_{216}$ cases, reported 
Table 1: Formation energies $\left(\mathrm{E}_{f}^{1}\right.$ and $\mathrm{E}_{f}^{2}$, in $\mathrm{eV}$ ) obtained with two different procedures (see Equation 3 and the text) for the B-center defect in diamond. Values obtained with different methods and basis sets refer to the $S_{64}$ supercells, while those for different supercells have been obtained at the B3LYP/6-21G level. The A-center formation energies ${ }^{29}$ have been reported for comparison.

\begin{tabular}{|c|c|c|c|c|}
\hline \multirow[b]{2}{*}{ Method } & \multirow[b]{2}{*}{ Basis Set } & \multicolumn{2}{|c|}{ B-center } & \multirow{2}{*}{$\begin{array}{c}\text { A-center } \\
\mathrm{E}_{f}^{29}\end{array}$} \\
\hline & & $\mathrm{E}_{f}^{1}$ & $\mathrm{E}_{f}^{2}$ & \\
\hline $\mathrm{HF}$ & 6-21 & 4.07 & 24.51 & 11.93 \\
\hline PBE0 & $6-21$ & 2.13 & 24.49 & 10.45 \\
\hline \multirow{4}{*}{ B3LYP } & $6-21$ & 2.95 & 22.41 & 9.70 \\
\hline & $6-21^{*}$ & 3.15 & 21.06 & 8.99 \\
\hline & $6-31$ & 4.17 & 22.23 & 9.51 \\
\hline & 6-31* & 3.66 & 20.15 & 8.45 \\
\hline HSE06 & $6-21$ & 2.11 & 24.46 & 10.43 \\
\hline $\mathrm{PBE}$ & $6-21$ & 2.26 & 23.47 & 9.79 \\
\hline LDA & $6-21$ & 0.50 & 24.80 & 9.77 \\
\hline$\overline{\overline{\text { Method }}}$ & Supercell & 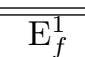 & 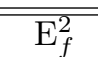 & \\
\hline \multirow{3}{*}{ B3LYP } & $\mathrm{S}_{216}$ & 2.95 & 22.40 & 9.80 \\
\hline & $\mathrm{S}_{512}$ & 2.95 & 22.41 & - \\
\hline & $\mathrm{S}_{1000}$ & 2.96 & 22.42 & - \\
\hline
\end{tabular}

in the two bottom panels (left and right, respectively) of Figure 2, shows that the energy dispersion of the defective levels, that in the former is about $1 \mathrm{eV}$, reduces to $0.35 \mathrm{eV}$ in the latter and even more, obviously, in $\mathrm{S}_{512}$ and $\mathrm{S}_{1000}$.

One of the most characterizing and interesting physical quantities related to a specific defect is, clearly, the corresponding formation energy. Starting from the pristine diamond structure (P) and four nitrogen atoms, the formation of the B-center defective structure (D) can be described as:

$$
\mathrm{P}+4 \cdot \mathrm{N} \rightarrow \mathrm{D}+5 \cdot \mathrm{C}
$$

This corresponds to a defect formation energy, $\mathrm{E}_{f}$, that can be written as follows

$$
\mathrm{E}_{f}=\left(\mathrm{E}_{\mathrm{P}}-5 \cdot \mathrm{E}_{\mathrm{C}}^{i}\right)-\left(\mathrm{E}_{\mathrm{D}}-4 \cdot \mathrm{E}_{\mathrm{N}}^{i}\right)
$$

where $E_{P}$ and $E_{D}$ are the total energies of the pristine and defective diamond supercells, while $\mathrm{E}_{\mathrm{C}}^{i}$ and $\mathrm{E}_{\mathrm{N}}^{i}$ are the energies of the isolated carbon and nitrogen atoms, respectively. 
The superscript $i=1,2$ indicates two different ways to calculate the atomic energies. When $i=1$, these energies are half of the total energy of the $\mathrm{N}_{2}$ molecule for nitrogen and the energy of a single carbon atom in the pristine diamond structure, whereas for $i=2$ they are the energies of the isolated atoms. Concerning the way $E_{\mathrm{C}}^{2}$ and $\mathrm{E}_{\mathrm{N}}^{2}$ are evaluated, a problem arises related to the tails of the atomic wavefunctions that have an exponential behavior, while the Gaussian-type functions decay to zero much faster. In order to correct for this wrong behavior, the Boys-Bernardi Counterpoise Correction, ${ }^{67}$ in which the basis functions of the neighboring atoms of the actual bulk system are included in the atomic calculation, is applied.

Since many calculated data about formation energies have been proposed in the literature, many times obtained with different computational methods with respect to B3LYP/6-21, in Table 1 formation energies computed with different Hamiltonians, spanning from HartreeFock to LDA, are reported for the $\mathrm{S}_{64}$ supercell. PBE, PBE0 and HSE06 functionals provide energies around $2.2 \mathrm{eV}$; B3LYP is about $0.7 \mathrm{eV}$ higher. $\mathrm{HF}$ and LDA, as usual, are at the extremes ( 4 and $0.5 \mathrm{eV}$, respectively). The effect of the defect concentration and of the basis set choice are also reported in the B3LYP case. The Table documents that the formation energy is already well converged at $\mathrm{S}_{64}$, whereas larger basis sets as $6-31 \mathrm{G}^{*}$ can further increase $\mathrm{E}_{f}$ by about $0.7 \mathrm{eV}$.

In all cases, however, the formation energy of the B center appears to be lower than the one obtained, with the same set of computational parameters and methods, for the A center: ${ }^{29}$ $3.31(2.79) \mathrm{eV}$ at the B3LYP (PBE0) level with the 6-21G basis set, to be compared to 2.95 (2.13) from Table 1. This behavior is due to the strong correlation energy of the $\mathrm{N}_{2}$ molecule, only partially taken into account by hybrid functionals; in the B-defect, containing $2 \mathrm{~N}_{2}$ units, the "correlation energy error" is twice the one of the A defect. If the formation energy of the defect is directly evaluated from the isolated atom energies $\left(\mathrm{E}_{f}^{2}\right)$, the problem is bypassed, and the obtained formation energies for the B defect are, roughly, twice the ones obtained for the A center ${ }^{29}$ (9.70 vs $22.41 \mathrm{eV}$ at the B3LYP 6-21G level). 
A reasonably accurate understanding of the relative concentration of the various defects, intrinsic and extrinsic, at various temperatures, would require a deeper analysis that should include vibrational contributions and the investigation of the many possible channels for diffusion from site to site, and then the accurate determination of the transition states along the lines explored in the relatively simple case of the I to $\mathrm{V}$ (self interstitial to vacancy) recombination. ${ }^{27}$ This analysis is deserved to a future study, as the main target of the present study is the IR spectroscopic characterization of the B defect.

\section{Spectroscopic characterization}

From the experimental side, it is generally rather difficult to assign spectral features to specific structural defects. This is not the case when starting from the simulation side, because in this case the kind of defect to be investigated and its concentration are defined a priori. We simulated the spectra of the B-defect and compared the results to experimental ones. We find some differences which could be due to many reasons, of which a considerable one is the possible presence of other defects in the experimental samples. This may lead to a superposition of signals.

The Raman spectrum of pristine diamond presents a single, sharp peak at $1332 \mathrm{~cm}^{-1}$; its simulated B3LYP/6-21G counterpart is at $1317 \mathrm{~cm}^{-1}$. The IR spectrum is even simpler: no signal at all in the full range of frequencies. If peaks appear in the IR spectrum of the defective system, they must then be attributed to the presence of some defects.

The computed Raman spectrum of the B center is characterized by the very intense "pris-

tine diamond" peak at $1317 \mathrm{~cm}^{-1}$, accompanied by a set of small peaks whose intensity is three to four orders of magnitude lower than the perfect diamond one. The B-center does not present then any interesting Raman feature; for this reason it will not be reported nor discussed furtherly.

In Figure 3 the IR spectrum of the defect at various concentrations is shown. In order to mimic the experimental broadening of the peaks, a linear combination of Lorentzian curves 


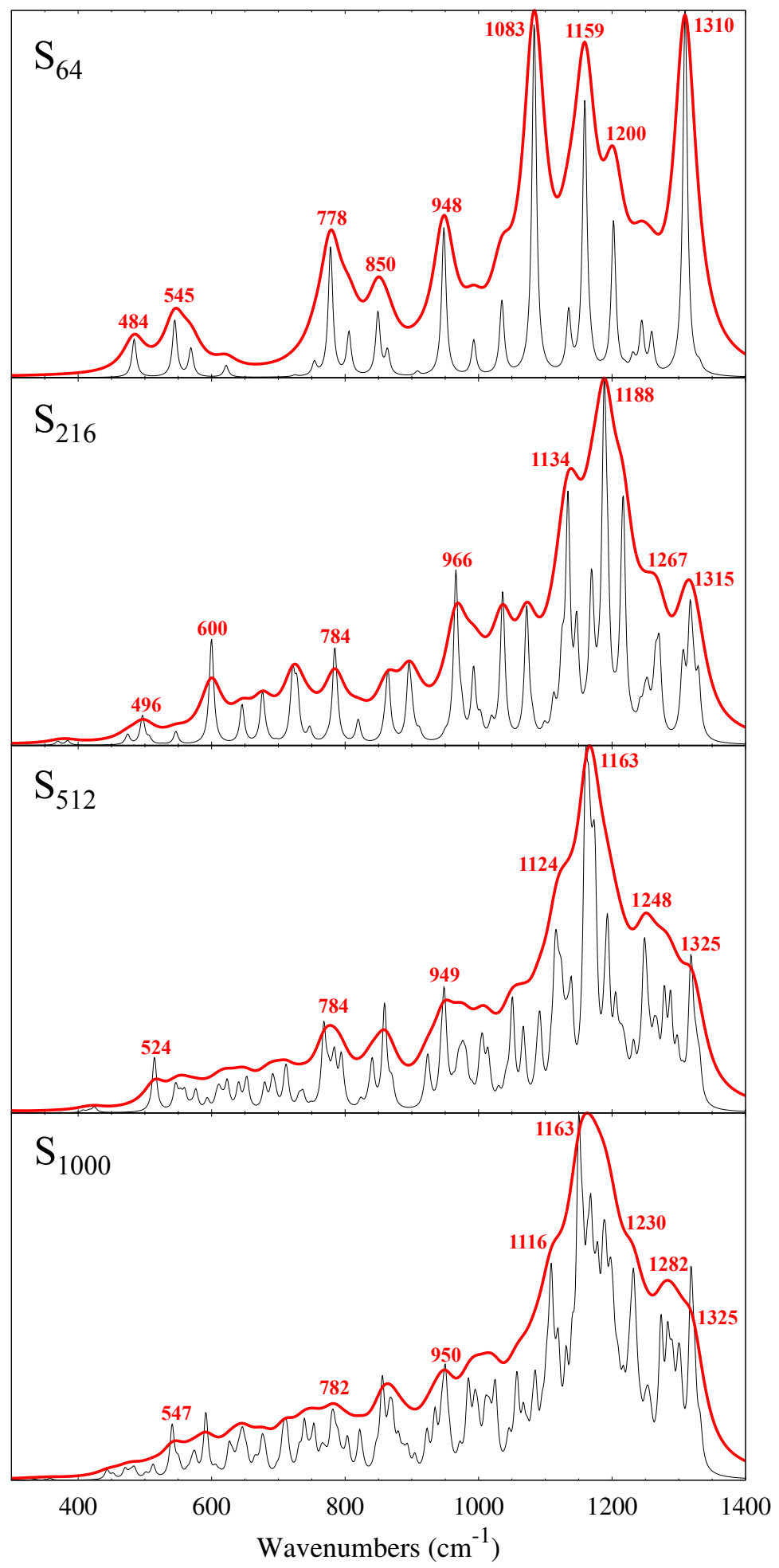

Figure 3: Simulated IR spectra of the B-center in diamond for the $\mathrm{S}_{64}, \mathrm{~S}_{216}, \mathrm{~S}_{512}$ and $\mathrm{S}_{1000}$ supercells. For each panel, two different values of full width at half maximum (FWHM) have been used: $8 \mathrm{~cm}^{-1}$ and $35 \mathrm{~cm}^{-1}$ for black and red curves, respectively. 
provides the pseudo-Voigt functional form; in particular, two different values of full width at half maximum of $8 \mathrm{~cm}^{-1}$ (black curve) and $35 \mathrm{~cm}^{-1}$ (red curve) have been used. We recall that all the selected supercells have the same cubic shape, so that the features of the spectra (peak positions and intensities) are only affected by the defect concentration. All the IR-active modes belong to the $t_{2}$ irreducible representation., The reported frequencies are not scaled.

The spectra can be divided in two regions, above and below $900 \mathrm{~cm}^{-1}$. The 900-1300

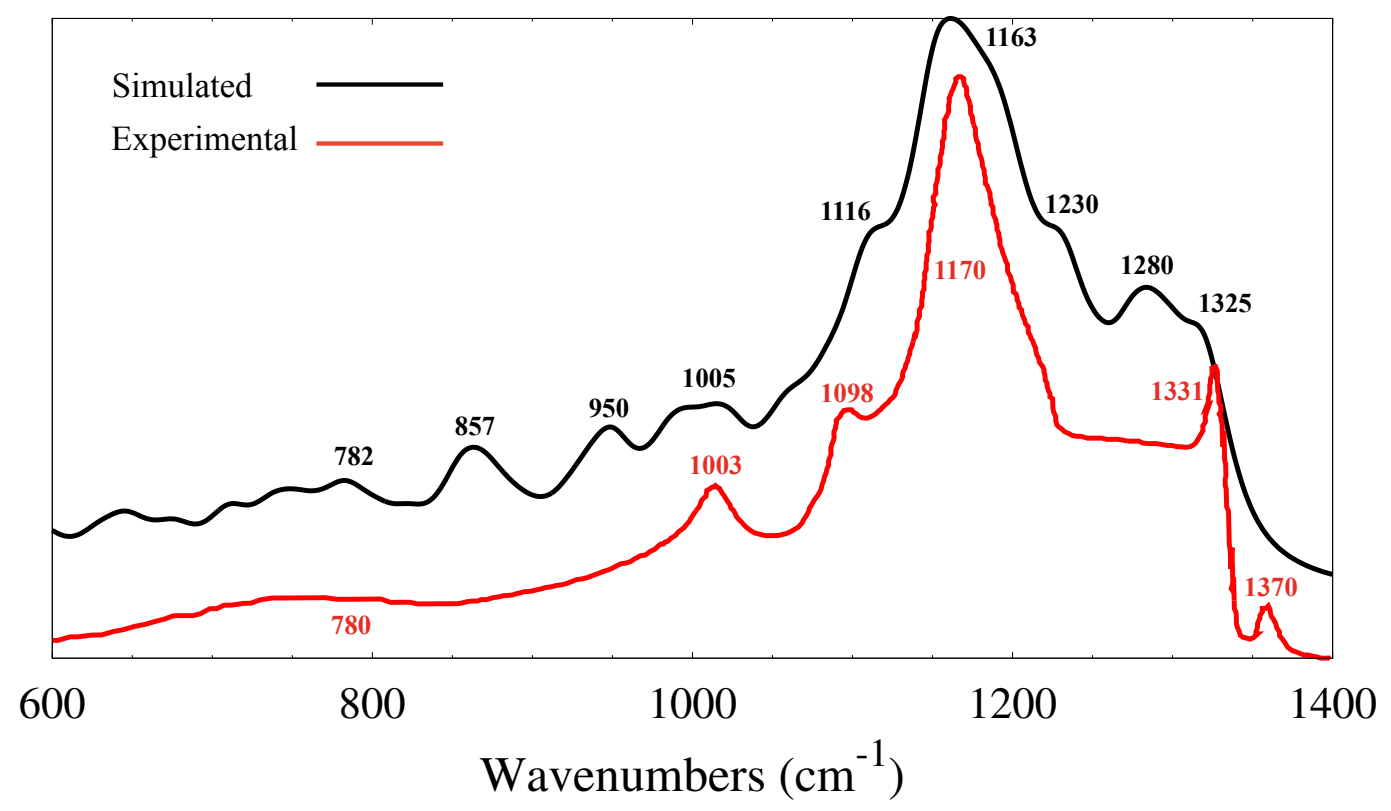

Figure 4: Comparison between experimental ${ }^{31,35}$ and simulated IR spectra of the B-center in diamond. The simulated spectrum, refers to the $\mathrm{S}_{1000}$ supercell. A FWHM of $35 \mathrm{~cm}^{-1}$ has been used.

$\mathrm{cm}^{-1}$ region is characterized by very intense peaks that, as the defect concentration lowers, progressively, merge into a central peak around $1160 \mathrm{~cm}^{-1}$. This peak, characterized by a large intensity, dominates the spectrum. Several less intense peaks appear as shoulders of the dominant peak up to $1330 \mathrm{~cm}^{-1}$. No peaks appear above $1330 \mathrm{~cm}^{-1}$.

The $900-400 \mathrm{~cm}^{-1}$ region is characterized by peaks with lower intensity with respect to those observed in the higher wavenumber region, and the overall shape of the spectra becomes smoother as the defect concentation reduces. As the Figure shows, the simulated spectra of 
$\mathrm{S}_{216}, \mathrm{~S}_{512}$ and $\mathrm{S}_{1000}$ show a similar structure, when large values of full width half maximum $\left(35 \mathrm{~cm}^{-1}\right)$ are used. Despite $S_{512}$ and $S_{1000}$ are rather large supercells, the two low energy regions of the spectra still show some differences. Vibrational normal modes involving the motions of all the atoms of the cell usually belong to this spectral region; therefore, the distance between defects belonging to neighboring cells (which passes from 14.41 to 18.01 $\AA$ ) might alter the profile of the spectrum. However, in the higher energy region, many of the spectroscopic features remain mostly unaltered when passing from $\mathrm{S}_{512}$ to $\mathrm{S}_{1000}$. It is important to underline that, despite several peaks appear in the 4 simulated IR spectra of the B-center reported in Figure 3, none of them is observed remarkably above the one-phonon absorption of the pristine diamond (being $1331 / 1332 \mathrm{~cm}^{-1}$ the experimental value), which means that vibrational normal modes involving a small amount of atoms are not present. As a further confirm, the animation of the vibrational modes show the lacking of localized vibrational normal modes.

The computed spectrum of $\mathrm{S}_{1000}$ has been compared with the experimental one in Figure 4. The latter (taken from Ref. 31,34) is not actually the whole spectrum of a synthetic or natural IaA/B diamond but only the B component decomposed according to different but similar schemes proposed by Davies, ${ }^{31}$ Clark and Davey ${ }^{35}$ and by Woods. ${ }^{32}$

The distinctive features of the experimental IR spectrum due to B-center aggregates are a spike of intensity around $1331 \mathrm{~cm}^{-1}$ (the Raman feature observed in the pristine diamond), a plateau between 1310 and $1230 \mathrm{~cm}^{-1}$, a maximum at $1170 \mathrm{~cm}^{-1}$ with a shoulder at 1098 $\mathrm{cm}^{-1}$, a less intense peak at $1003 \mathrm{~cm}^{-1}$ and a broad weak band around $780 \mathrm{~cm}^{-1}$. Out of the 7 wavenumbers proposed by Sutherland, ${ }^{1}$ the peaks at 328,1372 (visible in the experimental spectrum reported) and $1426 \mathrm{~cm}^{-1}$ were discarded as attributed to platelets. ${ }^{32,33}$ The peak observed at $1098 \mathrm{~cm}^{-1}$ was overlooked by Sutherland since masked by the band of A aggregates at the same frequency.

The shape of the experimental spectrum is globally reproduced by the simulated one, the main difference being the absence of the plateau between 1310 and $1230 \mathrm{~cm}^{-1}$. 
For the experimental peak at at 1170 , we find a corresponding peak at $1163 \mathrm{~cm}^{-1}$. The peak at $1098 \mathrm{~cm}^{-1}$, that appears as a shoulder in the experimental spectrum, can be observed at $1116 \mathrm{~cm}^{-1}$ in the simulated one. The experimental peak around the Raman frequency, 1331 $\mathrm{cm}^{-1}$, does not appear in the simulated spectrum as a clear spike as in the experimental case; concerning this aspect, the intensity of this peak seems to be correlated to the amount of vacancies in the structure. ${ }^{36,37}$

Moving to the peaks below $1100 \mathrm{~cm}^{-1}$, the spectroscopic feature reported by Sutherland at $1003 \mathrm{~cm}^{-1}$ is observed in our simulation at $1005 \mathrm{~cm}^{-1}$ ), while there is no mention in the experimental works ${ }^{1,31}$ of the simulated spectroscopic features around 950 and $857 \mathrm{~cm}^{-1}$. It is worthy to note that the peak around $780 \mathrm{~cm}^{-1}$ reported by Sutherland is invisible in the simulated spectrum when large supercells are considered $\left(S_{512}\right.$ and $\left.S_{1000}\right)$, while shows a remarkable intensity when higher defect concentrations are used $\left(\mathrm{S}_{64}\right.$ and $\mathrm{S}_{216}$, see Figure 3): this might be explained by a non homogeneous concentration of nitrogen defects in the experimental samples.

As a final remark, our simulation did not show any spectroscopic features around 400, 1370 and $1430 \mathrm{~cm}^{-1}$, confirming ${ }^{32,33}$ that these values observed by Sutherland ${ }^{1}$ are not B-center peaks.

\section{Conclusions}

The geometrical, electronic and spectroscopic features of the B-center in diamond have been investigated through a supercell scheme, as implemented in the CRYSTAL code. Four different supercells have been considered with 64, 216, 512 and 1000 atoms in the cell. The local structure of the defect is discussed in terms of the geometrical parameters and charge density distribution. The analysis of the band-structure of the B-center shows that the defect introduced an occupied level in the gap close to the valence band edge. The computed IR spectrum (wavenumbers and intensities) shows a large superposition with the experimental 
one, the main peaks at 780, 1003, 1098, 1171 and $1332 \mathrm{~cm}^{-1}$ being accurately reproduced. The high numerical accuracy and speed of the adopted code, that permit to afford the calculation of the vibrational frequencies and IR intensities of supercells containing up to 1000 atoms with hybrid functionals, is the key ingredient for an accurate simulation of the vibrational spectra of non simple defects like the B-center here investigated.

\section{Acknowledgements}

JKD would like to acknowledge funding from a Vanier Canada Graduate Scholarship.

\section{References}

(1) Sutherland, G. B. B. M.; Blackwell, D. E.; Simeral, W. G. Nature 1954, 174, 901-904.

(2) Davies, G. J. Phys. C 1976, 9, L537-L542.

(3) Breuer, S. J.; Briddon, P. Phys. Rev. B 1995, 51, 6984-6994.

(4) Mainwood, A. Diamond Relat. Mater. 1999, 8, 1560-1564.

(5) Kalish, R.; Reznik, A.; Prawer, S.; Saada, D.; Adler, J. Phys. Status Solidi A 1999, $174,83-99$.

(6) Davies, G.; Campbell, B.; Mainwood, A.; Newton, M.; Watkins, M.; Kanda, H.; Anthony, T. Phys. Status Solidi A 2001, 186, 187-198.

(7) Goss, J. P.; Coomer, B. J.; Jones, R.; Fall, C. J.; Briddon, P. R.; Öberg, S. Phys. Rev. B 2003, 67, 165208.

(8) Goss, J. P.; Jones, R.; Shaw, T. D.; Rayson, M. J.; Briddon, P. R. Phys. Status Solidi A 2001, 186, 215-220.

(9) Davies, G.; Smith, H.; Kanda, H. Phys. Rev. B 2000, 62, 1528-1531. 
(10) Kalish, R.; Reznik, A.; Nugent, K. W.; Prawer, S. Nucl. Instrum. Meth. Phys. Res. B 1999, $148,626-633$.

(11) Twitchen, D. J.; Hunt, D. C.; Newton, M. E.; Baker, J. M.; Anthony, T. R.; Banholzer, W. F. Physica B: Cond. Matter 1999, 273, 628-631.

(12) Lai, P. F.; Prawer, S.; Noble, C. Diam. Relat. Mater. 2002, 11, 1391-1396.

(13) Moroño, A.; de Vicente, S. M. G.; Hodgson, E. R. Fusion Eng. Des. 2007, 82, 25632566.

(14) Amekura, H.; Kishimoto, N. J. Appl. Phys. 2008, 104, 63509.

(15) Prawer, S.; Rosenblum, I.; Orwa, J. O.; Adler, J. Chem. Phys. Lett. 2004, 390, 458-461.

(16) Hyde-Volpe, D.; Slepetz, B.; Kertesz, M. J. Phys. Chem. 2010, 114, 9563-9567.

(17) Jamieson, D. N.; Prawer, S.; Nugent, K. W.; Dooley, S. P. Phys. Rev. B 1995, 106, 641-645.

(18) Hunn, J. D.; Withrow, S. P.; White, C. W.; Jr., D. M. H. Phys. Rev. B 1995, 52, 8106-8111.

(19) Prawer, S.; Nugent, K. W.; Jamieson, D. N. Diam. Relat. Mater. 1998, 7, 106-110.

(20) Orwa, J. O.; Nugent, K. W.; Jamieson, D. N.; Prawer, S. Phys. Rev. B 2000, 62, 5461-5472.

(21) Brunetto, R.; Baratta, G. A.; Strazzulla, G. J. Appl. Phys. 2004, 96, 380-386.

(22) Olivero, P.; Rubanov, S.; Reichart, P.; Gibson, B. C.; Huntington, S. T.; Rabeau, J. R.; Greentree, A. D.; Salzman, J.; Moore, D.; Jamieson, D. N.; Prawer, S. Diamond Relat. Mater. 2006, 15, 1614-1621. 
(23) Bergman, A. A.; Zaitsev, A. M.; Huang, M.; Gorokhovsky, A. J. Lumin. 2009, 129, $1524-1526$.

(24) Baima, J.; Zelferino, A.; Olivero, P.; Erba, A.; Dovesi, R. Phys. Chem. Chem. Phys. 2016, 18, 1961-1968.

(25) Zelferino, A.; Salustro, S.; Baima, J.; Lacivita, V.; Orlando, R.; Dovesi, R. Theor. Chem. Acc. 2016, 135, 1-11.

(26) Salustro, S.; Erba, A.; Zicovich-Wilson, C. M.; Nöel, Y.; Maschio, L.; Dovesi, R. Phys. Chem. Chem. Phys. 2016, 120, 21288-21295.

(27) Salustro, S.; Nöel, Y.; Zicovich-Wilson, C. M.; Olivero, P.; Dovesi, R. J. Chem. Phys. 2016, 145, 184701.

(28) Salustro, S.; Ferrari, A. M.; Orlando, R.; Dovesi, R. Theor. Chem. Acc. 2017, 4, 1-13.

(29) Salustro, S.; Sansone, G.; Zicovich-Wilson, C. M.; Noël, Y.; Maschio, L.; Dovesi, R. Phys. Chem. Chem. Phys. 2017, 19, 14478-14485.

(30) Loubser, J. H. N.; Wyk, J. A. V. Reading, UK.

(31) Davies, G. Nature 1981, 290, 40-41.

(32) Woods, G. S. Platelets and the Infrared Absorption of Type Ia Diamonds. Proceedings of the Royal Society of London A: Mathematical, Physical and Engineering Sciences. 1986; pp 219-238.

(33) Woods, G. S. Phil. Mag. Lett. 1989, 59, 339-342.

(34) Clark, C. D.; Davey, S. T. Phys. C Solid State 1984, 17, L399.

(35) Clark, C. D.; Davey, S. T. Phys. C Solid State 1984, 17, 1127-1140.

(36) Collins, A. T.; Spear, P. M. Phys. D. Appl. Phys 1982, 15, L183. 
(37) Evans, T.; Qi, Z.; Maguire, J. Journal of Physics C: Solid State Physics 1981, 14, L379.

(38) Goss, J. P.; Coomer, B. J.; Jones, R.; Shaw, T. D.; Briddon, P. R.; Rayson, M.; Öberg, S. Phys. Rev. B 2001, 63, 195208.

(39) Gali, A.; Maze, J. R. Phys. Rev. B 2013, 88, 235205.

(40) Katcho, N. A.; Carrete, J.; Li, W.; Mingo, N. Phys. Rev. B 2014, 90, 094117.

(41) Chanier, T.; Gali, A. Phys. Rev. B 2013, 87, 245206.

(42) Dovesi, R.; Orlando, R.; Erba, A.; Zicovich-Wilson, C. M.; Civalleri, B.; Casassa, S.; Maschio, L.; Ferrabone, M.; Pierre, M. D. L.; D'Arco, P.; Noël, Y.; Causà, M.; Rérat, M.; Kirtman, B. Int. J. Quantum Chem. 2014, 114, 1287.

(43) Becke, A. D. J. Chem. Phys. 1993, 98, 5648-5652.

(44) Lee, C.; Yang, W.; Parr, R. Phys. Rev. B 1988, 37, 785-789.

(45) Dirac, P. Proc. R. Soc. A 1930, 126, 360-365.

(46) Perdew, J. P.; Burke, K.; Ernzerhof, M. Phys. Rev. Lett. 1996, 77, 3865-3868.

(47) Adamo, C.; Barone, V. Chem. Phys. Lett. 1998, 298, 113-119.

(48) Krukau, A. V.; Vydrov, O. A.; Izmaylov, A. F.; Scuseria, G. E. J. Chem. Phys. 2006, $125,224106-224106$.

(49) Binkley, J. S.; Pople, J. A.; Hehre, W. J. J. Am. Chem. Soc. 1980, 102, 939-947.

(50) Francl, M. M.; Petro, W. J.; Hehre, W. J.; Binkley, J. S.; Gordon, M. S.; DeFrees, D. J.; Pople, J. A. J. Chem. Phys. 1982, 77, 3654-3665.

(51) Becke, A. D. J. Chem. Phys. 1988, 88, 2547-2553. 
(52) Dovesi, R.; Saunders, V. R.; Roetti, C.; Orlando, R.; Zicovich-Wilson, C. M.; Pascale, F.; Civalleri, B.; Doll, K.; Harrison, N. M.; Bush, I. J.; D’Arco, P.; Llunell, M. CRYSTAL 2014 User's Manual; ; University of Torino, Torino, 2013.

(53) Monkhorst, H. J.; Pack, J. D. Phys. Rev. B 1976, 13, 5188.

(54) C. Pisani and R. Dovesi and C. Roetti, Hartree-Fock Ab Initio Treatment of Crystalline Systems; Springer, 1988.

(55) Pascale, F.; Zicovich-Wilson, C. M.; Gejo, F. L.; Civalleri, B.; Orlando, R.; Dovesi, R. J. Comput. Chem. 2004, 25, 888-897.

(56) Zicovich-Wilson, C. M.; Pascale, F.; Roetti, C.; Saunders, V. R.; Orlando, R.; Dovesi, R. J. Comput. Chem. 2004, 25, 1873-1881.

(57) Erba, A.; Ferrabone, M.; Orlando, R.; Dovesi, R. J. Comput. Chem. 2013, 34, 346-354.

(58) Carteret, C.; De La Pierre, M.; Dossot, M.; Pascale, F.; Erba, A.; Dovesi, R. J. Chem. Phys. 2013, 138, 014201.

(59) Baima, J.; Ferrabone, M.; Orlando, R.; Erba, A.; Dovesi, R. Phys. Chem. Minerals 2016, 43, 137-149.

(60) Doll, K.; Saunders, V. R.; Harrison, N. M. Int. J. Quan. Chem. 2000, 82, 1-13.

(61) Barrow, G. M. Introduction to Molecular Spectroscopy; McGraw-Hill, New York, 1962; Chapter 4, p 70.

(62) Hess, B. A.; Schaad, L. J.; Carsky, P.; Zahradnik, R. Chem. Rev. 1986, 86, 709-730.

(63) Maschio, L.; Kirtman, B.; Orlando, R.; Rérat, M. J. Chem. Phys. 2012, 137, 204113.

(64) Maschio, L.; Kirtman, B.; Rérat, M.; Orlando, R.; Dovesi, R. J. Chem. Phys. 2013, 139, 167101. 
(65) Maschio, L.; Kirtman, B.; Rérat, M.; Orlando, R.; Dovesi, R. J. Chem. Phys. 2013, 139, 164101.

(66) Maschio, L.; Kirtman, B.; Rérat, M.; Orlando, R.; Dovesi, R. J. Chem. Phys. 2013, 139, 164102.

(67) Boys, S. F.; Bernardi, F. Mol. Phys. 1970, 19, 553-566. 